\title{
Relativistic Approximations for Quantization and Harmony in the Schrödinger Equation, and Why Mechanics Is Quantized
}

\author{
Antony J. Bourdillon \\ UHRL, San Jose, CA, USA \\ Email: bourdillona@sbcglobal.net
}

How to cite this paper: Bourdillon, A.J. (2020) Relativistic Approximations for Quantization and Harmony in the Schrödinger Equation, and Why Mechanics Is Quantized. Journal of Modern Physics, 11, 1926-1937. https://doi.org/10.4236/jmp.2020.1112121

Received: October 28, 2020

Accepted: December 6, 2020

Published: December 9, 2020

Copyright (๑) 2020 by author(s) and Scientific Research Publishing Inc. This work is licensed under the Creative Commons Attribution International License (CC BY 4.0).

http://creativecommons.org/licenses/by/4.0/

\section{(c) (i) Open Access}

\begin{abstract}
The initial purpose is to add two physical origins for the outstandingly clear mathematical description that Dirac has left in his Principles of Quantum Mechanics. The first is the "internal motion" in the wave function of the electron that is now expressed through dispersion dynamics; the second is the physical origin for mathematical quantization. Bohr's model for the hydrogen atom was "the greatest single step in the development of the theory of atomic structure." It leads to the Schrodinger equation which is non-relativistic, but which conveniently equates together momentum and electrostatic potential in a representation containing mixed powers. Firstly, we show how the equation is expansible to approximate relativistic form by applying solutions for the dilation of time in special relativity, and for the contraction of space. The adaptation is to invariant "harmonic events" that are digitally quantized. Secondly, the internal motion of the electron is described by a stable wave packet that implies wave-particle duality. The duality includes uncertainty that is precisely described with some variance from Heisenberg's axiomatic limit. Harmonic orbital wave functions are self-constructive. This is the physical origin of quantization.
\end{abstract}

\section{Keywords}

Wave Packet, Wave-Particle Duality, Harmony, Relativity, Dispersion Dynamics

\section{Introduction}

For the electron, Dirac's unspecified "internal motion", that is implied by relativity and quantum physics, is only fleetingly mentioned in his Principles [1]. That motion is here expressly analyzed in the wave packet, and this provides a 
clear explanation for wave-particle duality. The model of the packet opposes one kind of quantum theory that is axiomatic and mathematical, (including: quantization; uncertainty limits; creation and annihilation operations; a supposedly unstable wave packet-even in absence of Newtonian force; unspecified reduction of the packet; etc.), against physical quantum mechanics that is empirical (including: quantization due either to stable and harmonic wave functions as in Bohr's hydrogen atom; or to conservation of mass; of particles; of charge; of energy; of momentum with classical wave-particle uncertainties; combined with continuous reduction of packets that are stable in the absence of external force; etc. [2]-[7]). Physically, this second set of properties is more substantial than the alternative mathematical representation by probabilistic statistics. The two representations frequently clash, not only in the wave packet but equally in neutrino induced radioactive decay (see Vasiliev [8]), and also in uncertainty as will be discussed below.

There are moreover, more profound differences: in mathematics it is convenient to simplify problems by abstractly limiting analysis; empirical physics, by contrast, includes evolving possibilities whether implicit in current theory, or whether perhaps measurable with future technology (as in the decay) and theory. A complete empirical physic is unattainable and noumenal. Each method has its merit at different phases of development in various fields in physics.

This merit is partly psychological: mathematical tautologies carry undeniable certainties: as a simple example, the summation $2+2=4$ is certain because of number definition. Modulo $4,2+2=10$ is equally certain and for indistinguishable formal reasons. Sometimes postulates have extreme consequences: the imaginary number $i$ underpins wave theory [9] and the whole of modern physics. Mathematical theories are in principle hypothetical. On the other hand, physical quantum mechanics is denied that tautological simplicity: hypothesis is to be falsified by measured fact [10] [11]. All hypotheses are equally true until one is falsified. Side-by-side, contradictory theories are normal in physics provided they are each, in principle, falsifiable, otherwise, any of them is physically meaningless. Meaningless also are editorial "opinions" that are not specifically verified by sufficient fact or consistent theory. Theoretical evolution is never complete. Some examples of these features are described in what follows ${ }^{1}$.

Whereas mathematicians believe their axioms when they are consistent within restrictive incompleteness [12] [13]; in physics by contrast, only falsifiable propositions are admissable: they begin logically "true", and become unphysical upon ${ }^{1}$ In confirmation, A. Pais wrote that "It was [Einstein's] almost solitary conviction that quantum mechanics is logically consistent, but that it is an incomplete manifestation of an underlying theory in which an underlying objective reality is possible." [Niels Bohr's Times (1991) Oxford. ISBN 0-19-852049-2 p. 433]. Actually, in his EPR paper [Einstein, A., Podolsky, B. and Rosen, N., Phys. Rev. 47 777-780 (19350] Einstein held that Bohr's theory is incomplete. The latter had claimed that all that can be known about an electron is its wave function, particularly with regard to momentum and position. This seems an unlikely theory since Gödel's mathematical theorems on completeness and consistency in axiomatic systems [12] [13]. This footnote does not contribute to the debate so much as outline background for arguments given in the text. 
falsification ${ }^{2}$. In particular, Bohr's hydrogenic orbits were quantized by numbers in energetic series. Numerical quantization is an easy operation, but needs the physical model. Physically, the quantization is due to constructive interference, over time, in quantized and harmonic electronic wave functions. The physical model is not always given (e.g. intrinsic spin [3]); but the relativistic approximation to be described here is both mathematical and physical.

The Schrödinger equation is non-relativistic, but corrections can be made in various ways. By contrast, the well-known Klein-Gordon equation does include mass energy and is consistently represented in second order, as Lorentz covariant. Alternatively, any solution of the free Dirac equation [1] is, component-wise, a solution of the free Klein-Gordon equation. Electromagnetic interactions can be incorporated, forming the topic of scalar electrodynamics. Here we return to the simple inclusion of electromagnetism that is found in the Schrodinger equation, and include special relativity by incorporating the invariant event of harmonization. Approximate solutions at low relativistic energies can then be accounted more easily than occurs in a wide literature e.g. [14] [15].

\section{Wave-Particle Duality and Harmonic Quantization}

The physical wave packet replaces Dirac's mysterious "internal motion". The packet is the volume within which the energy of a photon or particle is contained: as in statistics, the normal shape is the Gaussian, in Figure 1. The dual wave-particle is described and explained by the stable wave packet $\phi$ :

$$
\phi=A \cdot \exp \left(\frac{X^{2}}{2 \sigma^{2}}+X\right) \text { with } X=i(\bar{k} x-\bar{\omega} t)
$$

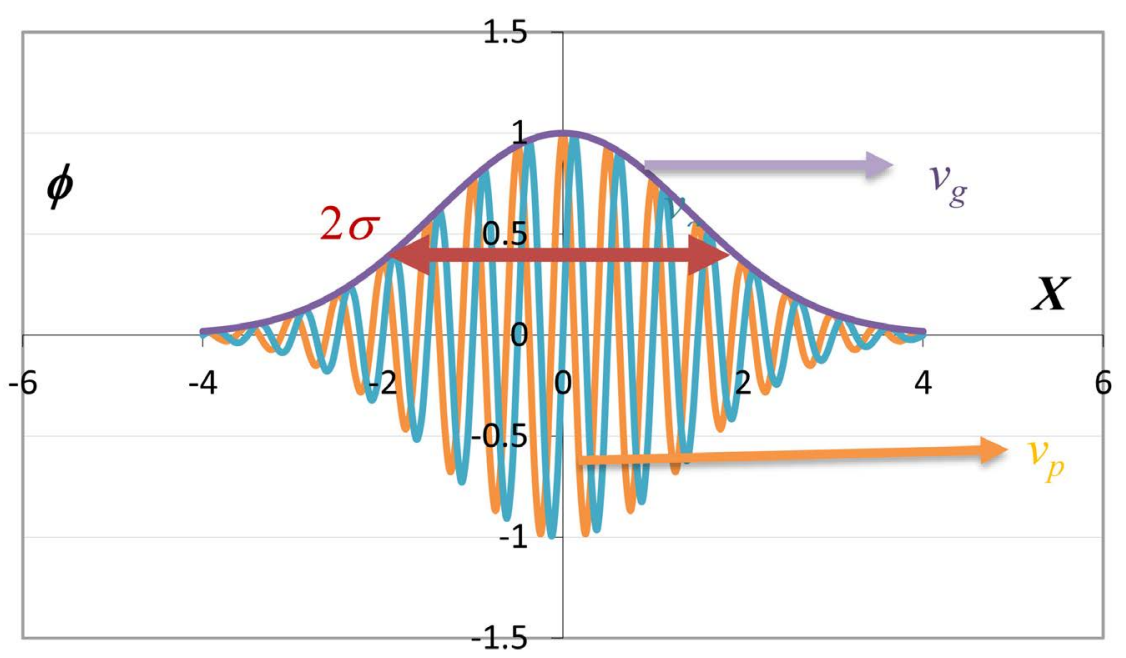

Figure 1. Stable wave packet (Equation (1)) containing envelope with group velocity $v_{g}$ (purple arrow) and real (orange) and imaginary (blue) parts of the carrier wave having phase velocity $v_{p}$ (horizontal orange arrow). The full width, at $\phi=1 / e$, is $2 \sigma$ as shown by the red double arrow.

${ }^{2}$ Physics verifies; Mathematics intuits; understanding unifies: "In the idea of the will, the real and ideal are united." [Hegel, G. W. F. (1969). Hegel s Science of Logic. Allen \& Unwin.] 
Its mean angular frequency $\bar{\omega}$ and mean wave vector $\bar{k}$, together, stabilize the free packet through conservation laws in energy and momentum. Here, the propagation is represented as unidirectional, while the two transverse directions may be represented by the normalized amplitude $A(x, y, z)$. This packet represents not only optical photons described by solutions to Maxwell's equations, but it represents equally the free electrons used in an electron microscope column for imaging, or for astronomic photons that have travelled billions of light years. The figure describes a complex carrier wave in a Gaussian envelope.

The frequency in this packet is given by Planck's Law and is proportional to energy $E$ :

$$
E=\hbar \omega
$$

that had been found quantized in photoemission from a bound atomic state. The reduced Planck constant is written $\hbar$. Bohr successfully applied this quantization to atomic spectra in the hydrogen atom: the wave orbitals harmonize as is represented provisionally on a planetary model (Figure 2) with red wave density amplitudes on a blue orbit.

In a particle, the displacements are not the real tensor fields known in electromagnetism; but components of a complex wave function. As Pauling observed, "[Bohr's] successful effort [at quantizing the spectrum of hydrogen], despite its simplicity, may be considered the greatest single step in the development of the theory of atomic structure [16]." Bound waves are harmonic on stationary states; otherwise the waves would self-annihilate. Of course, the constructive self-interference occurs on all three spatial dimensions (not just on the plane illustrated in Figure 2). The harmonization is provided in Schrödinger's curvilinear solutions to his equation, with three integral quantum numbers.

The free electron wave function is complex in Figure 1, but it is similar to quantized photons adapted from Maxwell's theory: the electromagnetic wave consists in two real components, an electric field $\boldsymbol{E}$ and lagging magnetic field $\boldsymbol{B}$ that is normal to both $E$ and the direction of propagation along the orbit. The phase lag between the two fields is $\pi / 2$ like the imaginary part of the particle wave function. The distinguishing difference is that the free electromagnetic

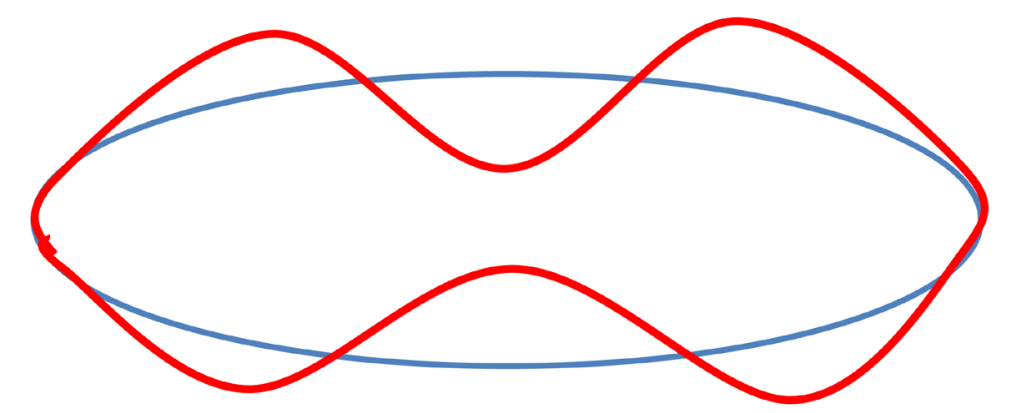

Figure 2. Schematic, 1-dimensional diagram showing three cycles of harmonic density functional red amplitude located on a blue orbit. The quantum number for this wave on this orbit is 4, but the full Schrödinger solution in curvilinear coordinates in 3-dimensions is more complicated [16]. 
wave has zero mass with group velocity equal to phase velocity, $v_{g}=v_{p}=c$, the speed of light in vacuo.

Prior to Bohr's success with quantized solutions for the hydrogen atomic states, Einstein had described the special theory of relativity: physical laws are invariant in all inertial reference systems. This includes the special case of $c$. There are several consequences that are summarized for the present discussion. In whatever inertial reference frame, the energy $E$, momentum $p$, and rest mass $m_{0}$, are related by Einstein's formula:

$$
E^{2}=\boldsymbol{p}^{2} c^{2}+m_{0}^{2} c^{4}
$$

which is often written in terms of relativistic mass $m$ !

$$
E=m^{\prime} c^{2}=\frac{m_{0} c^{2}}{\left(1-\beta^{2}\right)^{1 / 2}}
$$

with $\beta=v_{g} / c$. Applying Planck's law (Equation (2)) and de Broglie's subsequent hypothesis $\boldsymbol{p}=h_{i} / \lambda=\hbar \boldsymbol{k} \simeq h_{j} / \lambda_{i}$, with $j=x, y$ or $z$ Cartesian components. Equation (3) is transformed:

$$
\omega^{2}=\boldsymbol{p}^{2} c^{2}+m_{0}^{2} c^{4} / \hbar^{2}
$$

with derived values in dispersion dynamics [2] [3]:

$$
\frac{\mathrm{d} \omega}{\mathrm{d} k} \cdot \frac{\omega}{k}=v_{g} \cdot v_{p}=c^{2}
$$

including second derivative curvature, which is most easily derived in simplified units, $c=1=\hbar$ :

$$
\frac{\mathrm{d}^{2} \omega}{\mathrm{d} k^{2}}=\frac{1}{m_{\mathrm{eff}}}=\frac{a}{F}
$$

where $m_{\text {eff }}$ is a relativistic effective mass, equal to the ratio of force $F$ to acceleration $a$ in Newton's second law of motion. In consequence, since $F$ depends on a physical law and is invariant in all inertial reference systems $\mathrm{d}^{2} \omega / \mathrm{d} k^{2}<0 \supset a$ $<0$ i.e. reversed. (When, as occurs for conducting electrons in electrostatic crystal fields, the electron energy-dispersive curvature is negative, so also is the Coulombic acceleration). Equations (3)-(7) are the essential formulae in Dispersion Dynamics. They are generally consistent with the Klein-Gordon equation that is likewise frame invariant.

\section{Properties of the Wave Packet}

Consider Equation (1) as the product of two wave components and normalizer $\phi=A \phi_{1} \cdot \phi_{2}$. The envelope $\phi_{1}$ is real; the carrier wave $\phi_{2}$ is complex. The wave packet in Figure 1 combines three essentials of modern physics: the particle, the wave and relativity.

Firstly, the purple envelope group, $\phi_{1}$, carries the corpuscular properties (energy and momentum) that Newton claimed for light. These properties were extended to particles and supported quantized events in Plank's law and de 
Broglie's hypothesis. Optics of light and electrons are almost the same, bur with the noteworthy exceptions of finite mass in the latter, along with slower group velocities $v_{g}<c$.

Secondly, the green and orange complex oscillations, $\phi_{2}$, within the group cause the diffractive interference that was systematically described by Huygens, Fresnel, Fraunhofer et al. The oscillations also determine the harmonies that quantize stationary states established by Schrödinger's solutions for the hydrogen atom. Whereas the carrier wave determines the principal features of interference, diffraction etc.; the values of the real product $\phi_{2}(x, t) * \phi_{2}(x, t)=e^{0}$ are everywhere constant in all space and time, so that the oscillations carry neither energy nor momentum: the frequency spectrum in $\phi_{2}$ has no influence on the stability of the wave group envelope, $\phi_{1}$, though the energy and momentum of the packet do indeed depend on its frequency $\omega$ and wave-vector $k=2 \pi / \lambda$. Meanwhile, the oscillations cause the harmonization of stationary carrier waves, at orbits or boundaries, during emission or absorption. This is the consequence of constructive self-interference.

Thirdly, the wave properties of $X$ in these functions are subject to the relativistic invariance of physical laws in all inertial reference systems. Equations (3)-(7) are some of its consequences. Figure 1 and Equation (1) describe the principal qualities of free particles (whether having zero or finite rest mass) in quantum physics.

Group and phase velocities of the relativistic wave functions are plotted elsewhere [2] [3]. The absolute value of the carrier wave $\phi_{2}$ is constant with $x$ and $t$, energy transport is described by its envelope $\phi_{1} .{ }^{3}$ The elastic union between the carrier wave $\phi_{1}$, and group envelope $\phi_{2}$, is a significant characteristic of wave-particle duality. The wave packet is the physical explanation for the dual phenomena.

\section{Uncertainty and Stability}

In axiomatic and mathematical quantum theory [1] built on quantized, stationary states $\phi(\bar{k}, \bar{\omega})$, Heisenberg's Uncertainty Principle (HUP) is the axiom used to describe transition relationships in energy and time, or in momentum and space: $\Delta p_{i} \cdot \Delta x_{j}=(\hbar / 2) \delta_{i j}$ where $i, j=0,1,2$ or 3 in four dimensions, and $\delta_{i j}$ is the Dirac delta. The usage is not universal: some authors omit the HUP [17]; others criticize it [18]. In Dispersion Dynamics by contrast, the uncertainties inherent in wave-particle duality are concisely and precisely described at an elementary physical level: on the central, rest frame $\left(m^{\prime}=m_{0}, X=0\right)$ in cases $X=0$, Equation (1) yields, by direct inspection, the component uncertainty $\Delta t=2 \sigma_{x}^{2}$ and by Fourier transform, $\Delta \omega=2 / \sigma_{x}^{2}$, since the Fourier transform of a Gaussian is Gaussian. The combined uncertainty, $\Delta t \cdot \Delta \omega=4$, i.e. independent of $\sigma_{x}$. Likewise, for cases $t=0, \Delta x=2 \sigma^{2}$ and $\Delta k_{x}=2 / \sigma_{x}^{2}$ and $\Delta x \cdot \Delta k_{x}=4$. The joint Uncertainties are 8 times greater than the extreme limit in the equivalent ${ }^{3} \mathrm{~A}$ simple example is the bow wave excited by a speedboat. 
HUP, i.e. after generalizing dimensions using Equation (2) etc. As examples, consider uncertainties, at various heights, that are accurately calculated about the near field for the case of Fresnel diffraction of electrons through a narrow slit (Table 1). Figure 3 is an aerial image [19] [20].

Notice firstly that by Newton's first law of motion, "Any particle continues in a state of rest or uniform motion in a straight line except in so far as it is compelled by applied external force to change that state." This law is true in his corpuscular theory, and it is true for the plane-wave, incident beam before entering the thin slit. This acts as an external force that changes the transverse uncertainty in a systematic way. It is not necessary to cite an uncertain HUP to describe how this happens; the stable wave packet in conventional wave optics provides the simple, methodical explanation [2] [6]. Indeed the stable wave packet itself provides the uncertainty "limit" that is itself uncertain in HUP. The derivation was given in the previous paragraph, and can be written in normal 4-dimensional relativistic notation:

$$
\Delta k_{i} \cdot \Delta x_{j}=4 \delta_{i j}, \text { where } i, j=0,1,2 \text { or } 3
$$

This is a more precise value than is given in the HUP. It can be generalized to terms of energy and momentum by multiplication by the constant $\hbar$, while the limit itself fundamentally physical: it is the immediate consequence of wave-particle duality.

Table 1. Various uncertainties illustrated in Figure 3, with explanations in legend. Notice anomalous uncertainties at the Critical Condition in column c. The $x$-direction is downwards.

\begin{tabular}{|c|c|c|c|c|}
\hline Uncertainty & a & b & c & d \\
\hline$\omega$ & $m^{\prime} c^{2} / \hbar$ & & & \\
\hline$\Delta \omega$ & $2 e / \hbar \quad \mathrm{rad} / \mathrm{s}$ & $<--$ & $<--$ & $<--$ \\
\hline$\Delta t$ & $4 \hbar / 2 e \quad \mathrm{~s}$ & $<--$ & $<-$ & $<--$ \\
\hline$k_{x}$ & $2 \pi / \lambda$ & & & \\
\hline$\Delta k_{x}$ & $\Delta \omega / V_{g} \quad \mathrm{~m}^{-1}$ & $\sim<--$ & $\sim<--$ & $\sim<--$ \\
\hline$\Delta x$ & $4 / \Delta k_{x} \quad \mathrm{~m}$ & $\sim<--$ & $\sim<--$ & $\sim<--$ \\
\hline$\Delta k_{y}$ & 0 & 0 & $-6 / s$ & $8 s / G \lambda$ \\
\hline$\Delta y$ & $\infty$ & $2 s$ & $2 s / 3$ & $\sim G \lambda / 2 s$ \\
\hline$\Delta k_{z}$ & 0 & $<--$ & $<--$ & $<-$ \\
\hline$\Delta z$ & $\infty$ & $<-$ & $<--$ & $<--$ \\
\hline Dual uncertainty & & & $\Delta k_{y} \cdot \Delta y \simeq-4$ & $\Delta k_{y} \cdot \Delta y \simeq 4$ \\
\hline
\end{tabular}

Explanatory notes: Column a, line 1: in simple units, $\omega$ is the relativistic mass, i.e. $m_{0}+$ kinetic energy; line 2: energy line width in the electron microscope; line 3: from dual uncertainty (Equation (8)); line 4: de Broglie hypothesis; line 5: From relativity (Equation (5)) $\omega \mathrm{d} \omega=k \mathrm{~d} k c^{2} ; \Delta k=\Delta \omega \frac{\omega}{k c^{2}}=\frac{\Delta \omega}{v_{g}} ;$ line 6: From wave dual uncertainty. Column c, line 7: The critical condition $k c^{2} v_{g}$; line 8: From wave dual uncertainty in this case abnormally small. Column d, line 9: zero in far field diffraction; line 10: from wave dual uncertainty. 


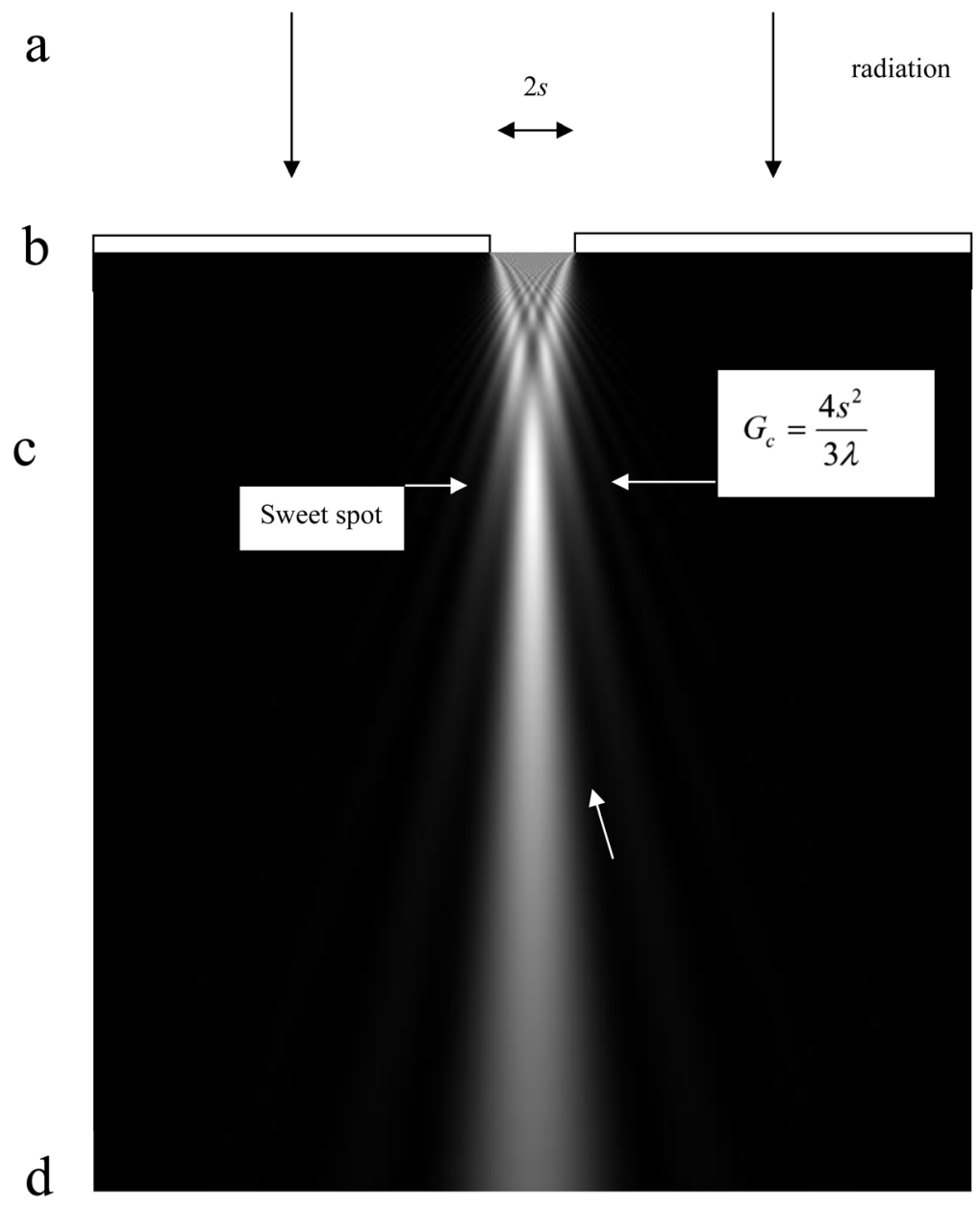

Figure 3. Aerial image calculated for an incident parallel incidence (level a) after emergence of a $100 \mathrm{KV}$, wavelength $\lambda$, electron beam from a slit, of width $s$, in a gold mask $10 \mu \mathrm{m}$ thick (level b) [19] [20]. Notice the focusing effect at the "Critical Condition".

Wave uncertainty not only complements the fact of wave-particle duality; it is more precise than the HUP and includes otherwise anomalous situations such as the Critical condition in near field. Dual wave uncertainty is also necessary in engineering applications [20], where precision has more value than mathematical oversimplification.

A further example of the increased precision is the fact that the wave optics demonstrates, through the wave function $X(k, x, \omega, t)$, a necessary relation between transverse $\sigma_{y}$ and $\sigma_{z}$, with $\sigma_{|k|}$ in the direction of propagation. This relation is neglected in the HUP but is exemplified in Table 1, cell a5. Many other examples of precision are predicted in the wave particle: the amplitude $A$ depends on the divergence of $\sigma$, expressed vectorially; the transverse wavelength $\lambda_{y}$ lengthens as the beam spreads, etc.

Moreover, the table illustrates the various ways in which the free wave packet is stable; but becomes partly unstable by the presence of applied external forces such as the narrow slit in Figure 3. Wave optics supplies a degree of determina- 
cy, within limits provided by wave particle duality. Experimental evidence requires it, for example as the condition' for proximity lithography (level c), and also in far field (level d), where the horizontal intensity profile approaches the Gaussian distribution. Precise uncertainty is a core property in wave-particle duality.

Notice further in Table 1 , that when $x>G_{o}$ the critical gap, then $\Delta y \cdot \Delta k_{y}>\hbar / 2 \pi$ which is divergent; but when $x<G_{\circ} \Delta y \cdot \Delta k_{y}<0$ and convergent. By contrast, the HUP is indiscriminate and inaccurate.

In concluding this section, notice that Heisenberg's principle correctly interprets the particle as a field, but his estimate is excessively restrictive when compared with known photon and electron optics. In orbital motion, the uncertainty is restricted by harmonies in the wave function. In measurement by reduction of the wave packet, (e.g. absorptive scintillation events in an interference pattern) multiple restrictions apply from sensing or emitting equipment. The HUP shows that "point particles" [3] are actually extended, and that the Copenhagen probabilistic theory of the wave function is mathematical, not physical

\section{Limitations of the Schrodinger Equation}

Compare with Equation (3) the Schrödinger equation in 3-dimensions which may be written for a system of $j$ particles bound to a central potential $V$ [16]:

$$
\sum^{N} \frac{\hbar^{2}}{2 m_{j}} \nabla_{j}^{2} \Psi-V \Psi_{j=1}=-i \hbar \frac{\partial \Psi}{\partial t}
$$

where $\nabla^{2}$ represents the Laplacian for the $t$ th particle, and the first term represents the summation of kinetic energy of the interacting particles; the second represents their electrostatic potentials; and the third term their energy $W_{m}$, or eigenvalues when expressed in matrix form for various quantized states. The equation is complementary to the free particle, 3-dimensional Equation (1) when its amplitude $A$ is small; $\sigma$ is large; and the function is made harmonic. In particular $\left\langle\phi^{*}\left|\frac{\partial}{\partial x}\right| \phi\right\rangle \simeq i k_{x}$, or $i p_{x} / \hbar$; while $\left\langle\phi^{*}\left|\frac{\partial}{\partial t}\right| \phi\right\rangle \simeq-i \omega$, or $-i E / \hbar$. However, equation (9) is not consistent with Equation (3): in Schrödinger's equation the masses $m_{i}$ are treated as constant and disregarded, and this is typically applied approximately in calculations of atomic structure. In relativity by contrast, particle masses vary with rest frame, and they sometimes absorb the major part of the kinetic energy: $E=m^{\prime} c^{2}=m_{0} c^{2} /\left(1-\beta^{2}\right)^{1 / 2}$, where $\beta=v_{g} / c$. Moreover, in relativity (Equation (3)) the three variables occur regularly in powers of 2, while the potential energies must be addedconsistently with Equation (9). Thus:

$$
E_{n}=\hbar \omega_{n}=W_{n}^{\prime}+m_{0} c^{2}
$$

where $W^{\prime}$ requires correction for relativistic contraction in space, with increasing $\beta$, and for dilation in time. Both $E_{n}$ and $W_{n}$ are functions of momentum, and therefore vary with rest frame. These features are implied in the Klein-Gordon equation. 
By contrast, Dirac factorized Equation (3) into two first order equations designed to operate on rank 4 matrix eigenvectors [1]. His antiparticles have negative rest mass and positive kinetic energy, which is problematic when $k=-m_{0} c$, since solutions for $\omega$ and $k$ become singular. Moreover, he uses Heisenberg dynamics to claim the speed of the electron equal to $c$, and this contradicts special relativity. Given the facts inherent in Dispersion Dynamics, we explore a different path that uses the advantage of quantized and harmonic self-constructive orbits (Figure 2) that are invariable events in any relativistic frame.

A paradigm solution that is enabled by these equations is the relativistic correction for eigenvalues of the Schrodinger equation. Calculate initially, the electronic, non-relativistic, solution using a preferred method such as the linear combination of atomic orbitals; Slater orbitals; hydrogenic wavefunctions etc. For illustration, consider hydrogenic functions. Treat the relativistic state as a perturbation on the non-relativistic state. We will account for changes in eigenvalues and eigenstates with length contractions, time dilations, and expectation values for momentum, velocity, mass etc.

The hydrogenic eigenvalues $W_{n}$ for quantum states $n$ are given by [16]:

$$
W_{n} \simeq \frac{\mu Z^{2}}{\hbar^{2}} \frac{e^{4}}{n^{2}}=\frac{Z^{2}}{a_{0}} \frac{e^{2}}{n^{2}}
$$

for electrons with rest mass $m_{e}$ and charge e orbiting nuclear charge $Z$. The Bohr radius $a_{0}=\hbar^{2} /\left(\mu e^{2}\right)$; and principal quantum number is $n$; where for atomic number $N$ and nuclear rest mass $m_{N}$, the reduced mass for an electron is:

$$
\mu=\frac{m_{e} \cdot m_{N}}{m_{e}+m_{N}} \simeq m_{e} \quad \text { when } m_{N} \gg m_{e}
$$

Notice that $W_{n}$ depends on the inverse of the Bohr radius which has the dimension of length:

$$
W_{n}=\frac{2 Z^{2} e^{2}}{a_{0} n^{2}}=\hbar \omega_{n}
$$

Then, the relativistic radial contraction corresponds to wavelength shortening and frequency growth: as time dilates, frequency shifts blue.

$$
a^{\prime}=a_{0}\left(1-\beta^{2}\right)^{1 / 2} ; \omega_{n}^{\prime}=\omega_{n}\left(1-\beta^{2}\right)^{-1 / 2}
$$

From these results are found approximate mean radii $\left\langle r_{n}\right\rangle$ for hydrogenic wavefunctions, and corresponding eigenvalues $W_{n}^{\prime}$ for states $n$.

In the non-relativistic approximation, the frequency is $W_{n} / h$ and phase velocity for the mean orbit is $v_{p}=2 \pi a_{0} W_{n} / h$. In relativity, $\beta=v_{p} / c=c / v_{p}$ for a free particle, so that $v_{g}=\hbar c^{2} /\left(a_{0} W_{n}\right)$, i.e. independent of $\beta$, in an observer frame, because fixed by the harmonic nature of the hydrogenic motion. ${ }^{4}$

${ }^{4}$ More generally, Equation (3) and Equation (5) are related: $E_{n}^{\prime 2}=W_{n}^{\prime 2}+m_{0}^{2} c^{4}=\left(K^{\prime}+V^{\prime}\right)^{2}+m_{0}^{2} c^{4}$ where again the primes indicate relativistically corrected values even though some are independent of rest frame, while $V$ can be expressed by the vector potential $A$. In case $W_{n} \ll m_{0} c^{2}$, the virial theorem gives, typically for a ground state, $W_{n} \simeq V^{\prime} / 2$, where the potential is negative. Then $k^{2} / 2 m_{0} \simeq-V / 2$, 


\section{Reactions to force in Crystal Fields}

In Dispersion Dynamics, as an immediate consequence of special relativity, particles react to force fields by accelerations that follow Equation (7): the force and acceleration are negative in negative dispersive curvature and vice versa. Electrons moving in negative curvature are often called "holes", and these are typically observed by positive coefficients in the Hall effect, most notably in high temperature superconductors [2] [5] (HiTc). The Hall effect demonstrates fundamental dynamic effects in electrons accelerated by electric crystals fields and applied magnetism. In superconductors, Dispersion Dynamics provides further explanation for extraordinary conduction where physical forces seem, prima facie, to disappear: inside the superconductor, the electric field $E=0$ because of zero resistivity; the magnetic field (in a type I superconductor) $\boldsymbol{B}=0$ by the Meissner-Ochsenfeld effect; $\boldsymbol{P}=0$ [21] [22] the momentum of the Cooper pair; the charge in the high temperature superconductor $Q=0$ in the Wannier excitons; and the spin $S=0$, because the positive Hall coefficients in HiTc shows the paired electrons are s-like and orthonormal. What then is left for the dynamics of the measured supercurrent? We suppose that the wave packet is reduced in Newtonian time by energy injection at electrodes so releasing electrons formerly bound into pairs by the superconducting gap [2].

More generally in condensed matter, relativistic effects become increasingly significant as binding energies or transport energies approach the rest mass energy of the electron $m_{e} c^{2}$. This occurs in deep core states in heavy atoms and in electron microscopes.

\section{Conclusion}

Given wave-particle duality that exists in the wave packet, harmonic events in a self-constructive wave function are Lorentz covariant and therefore subject both to normal time dilation in special relativity, and to space contraction. Atomic orbital radii and eigenvalues, such as those derived from the Schrödinger equation, can be used in approximation to account for relativistic effects in spatial and temporal accounting over a range of physical properties. These are significant at energies that approach the rest mass energy of the electron, as in core state scattering of electrons in EXAFS (extended X-ray absorption fine structure) from heavy elements, and in electron microscopes, especially in high energy instruments used for imaging thick or heavy specimens. The relativistic approximations are comparatively simple. They add many applications, previously described, for dispersion dynamics that are derived from the wave packet in special relativity. The applications add physical consistency to mathematical axioms that are chosen to represent quantization in energy states, sometimes with uncertain approximations.

\section{Conflicts of Interest}

The author declares no conflicts of interest regarding the publication of this paper. 


\section{References}

[1] Dirac, P.A.M. (1958) Principles of Quantum Mechanics. 4th Edition, Oxford University Press, Oxford.

[2] Bourdillon, A.J. (2017) Dispersion Dynamics in the Hall Effect and Pair Bonding in $\mathrm{HiT}_{\mathrm{c}}$. Nova Science, Hauppauge.

[3] Bourdillon, A.J. (2018) Journal of Modern Physics, 9, 2295-2307. https://doi.org/10.4236/jmp.2018.913145

[4] Bourdillon, A.J. (2018) Journal of Modern Physics, 9, 1304-1316. https://doi.org/10.4236/jmp.2018.96079

[5] Bourdillon, A.J. (2017) Journal of Modern Physics, 8, 483-499. https://doi.org/10.4236/jmp.2017.84031

[6] Bourdillon, A.J. (2015) Journal of Modern Physics, 6, 2011-2020. https://doi.org/10.4236/jmp.2015.614407

[7] Bourdillon, A.J. (2015) Journal of Modern Physics, 6, 463-471. https://doi.org/10.4236/jmp.2015.64050

[8] Vasiliev, B. (2020) Journal of Modern Physics, 11, 1263-1278. https://doi.org/10.4236/jmp.2020.116055

[9] Wittgenstein, L. (1956) Remarks on the Foundations of Mathematics. Tr. Anscombe, G.E.M., MIT, Cambridge.

[10] Popper, K.R. (1959) The Logic of Scientific Discovery. Hutchinson, London. https://doi.org/10.1063/1.3060577

[11] Popper, K.R. (1982) Quantum Theory and the Schism in Physics. Hutchinson, London.

[12] Gödel, K. (1931) Monatshefte für Mathematik und Physik, 38, 173-198. https://doi.org/10.1007/BF01700692

[13] Nagel, E. and Newman, J.R. (1959) Gödel's Proof. Routlegde \& Kegan Paul, Abingdon-on-Thames.

[14] Devian, C. and Bertrand, J. (2020) Journal of Modern Physics, 11, 1263-1278. https://doi.org/10.4236/jmp.2020.119079

[15] De Peralta, L. (2020) Journal of Modern Physics, 11, 788-802. https://doi.org/10.4236/jmp.2020.116051

[16] Pauling, L. and Wilson, E.B. (1935) Introduction to Quantum Mechanics. McGraw-Hill, New York.

[17] Isaacs, A. (1990) Concise Dictionary of Physics. Oxford University Press, Oxford.

[18] Olszewski, S. (2015) Journal of Modern Physics, 6, 22-626. https://doi.org/10.4236/jmp.2015.65067

[19] Bourdillon, A.J., Boothroyd, C.B., Kong, J.R. and Vladimirsky, Y. (2000) Journal of Physics D: Applied Physics, 33, 2133. https://doi.org/10.1088/0022-3727/33/17/307

[20] Bourdillon, A.J. and Vladimirsky, Y. (2006) X-Ray Lithography-On the Sweet Spot. UHRL, San Jose.

[21] Bardeen, J., Cooper, L.N. and Schrieffer, J.R. (1957) Physical Review, 106, 162-164. https://doi.org/10.1103/PhysRev.106.162

[22] Bardeen, J., Cooper, L.N. and Schrieffer, J.R. (1957) Physical Review, 108, 1175-1204. https://doi.org/10.1103/PhysRev.108.1175 